\title{
ELECTRODYNAMICS OF THE CENTRAL REGIONS OF ACTIVE GALACTIC NUCLEI
}

\author{
M. Salvati \\ Osservatorio Astrofisico di Arcetri \\ L. E. Fermi 5 \\ 1-50125 Firenze \\ Italy
}

\begin{abstract}
We review the information available on some bulk properties of the non-thermal emission regions in Active Galactic Nuclei, such as streaming motions, nature of composing particles, brightness temperature in the proper frame. Most of the information concerns the compact radio emission at relatively large distances from the center, whereas most of the action is thought to occur in the innermost regions which are observed at higher frequencies. We investigate whether these bulk properties can be understood within a general scheme for energy transport and particle acceleration around a magnetized rotating body.
\end{abstract}

\section{INTRODUCTION}

Investigating the electrodynamics of Active Galactic Nuclei (AGN's) by means of radio observations has an inherent difficulty. This is illustrated in Fig. 1, which shows a composite spectrum of the QSO 3C273 in the form of a plot of $v \mathrm{~F}_{y}$-i.e., flux per logarithmic frequency interval - versus $v$; plots of this kind give an indication on how the total power is distributed among the various spectral domains. The distribution is remarkably flat from the infrared up to the gamma rays; the exception is represented by the radio frequencies, whose contribution to the total luminosity is only a minor one.

The situation has been summarized by saying that, as far as the overall AGN emission is concerned, the radio emission is just "smoke", and the "fire" has to be looked for at higher frequencies. In the present paper we address precisely the problem of learning about the "fire" by studying the "smoke".

\section{RELATIVISTIC MOTIONS AND COMPACTNESS}

For our purposes, the two most important pieces of information derived from radio studies of AGN's are superluminal motions and rapid flux variations. By 


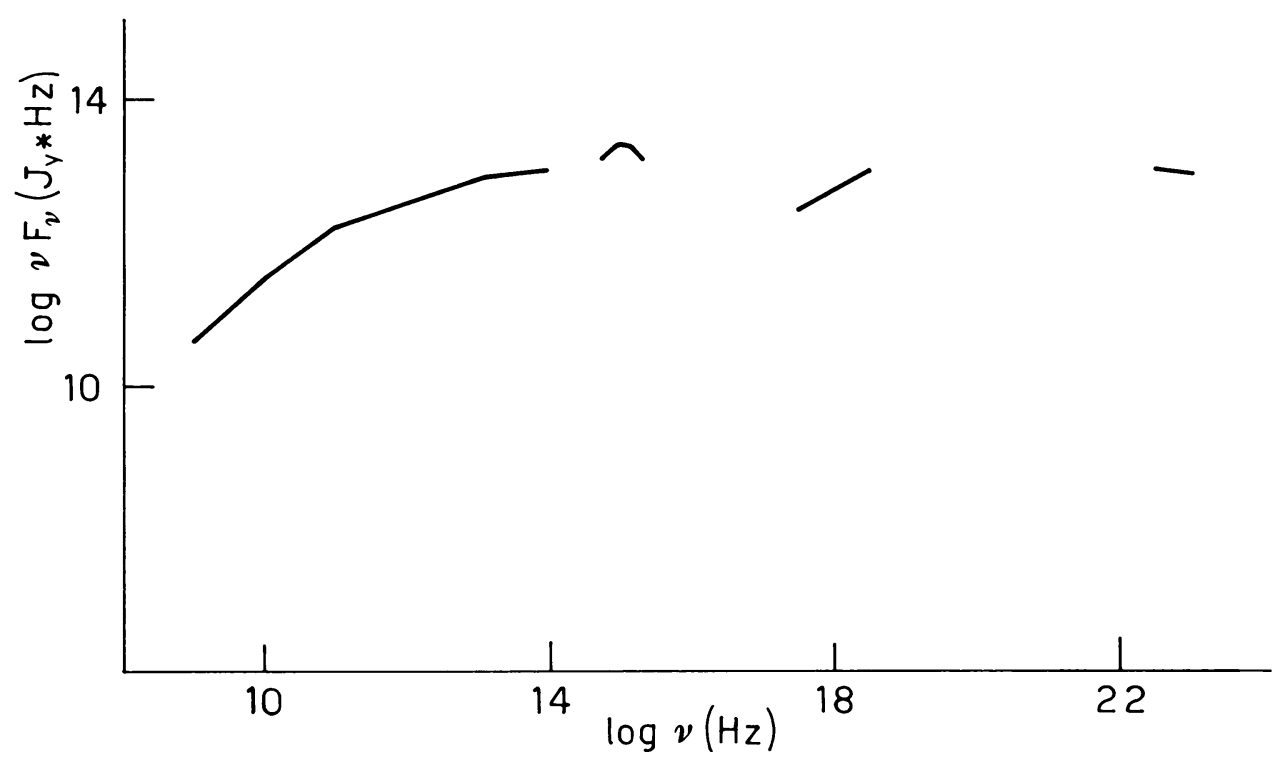

Figure 1. Composite spectrum of $3 \mathrm{C}_{273}$, showing the relative contribution of the various spectral regions to the total luminosity.

superluminal motions one means angular displacements of milliarcsec radio features, which imply faster-than-light transverse velocities at the distances indicated by the optical redshifts; these displacements have been firmly established by modern VLBI techniques, and cannot be explained away as due to insufficient sampling of the Fourier plane (Cohen and Unwin 1984).

Among the numerous interpretations which have been put forward, the one which encounters the largest degree of consensus involves the bulk motion of the emitting plasma at a speed very close to the speed of light along a trajectory very close to the line of sight (Blandford et 81.1977 ). This approach has some difficulties of its own, and its decisive advantage over the competing ones is that it provides a powerful unifying scheme for several other phenomena (Begelman et al.1984).

Additional evidence for the occurence of relativistic bulk motions in AGN's derives from rapid flux variability. The observational situation is rather complicated, especially at low radio frequencies where the importance of propagation effects in the interstellar medium has been recognized only recently (Padrielli 1984; Rickett 1986). At any rate, in at least some instances the observed variations appear to be intrinsic to the source; then, using the well-known causality argument to infer the source size $r$ from the variability time scale $\Delta t, r<c \Delta t$, one runs into serious difficulties in building up a consistent synchrotron model.

In their simplest form, models of this kind require only a few ingredients (see, e.g., Rybicki and Lightman 1979). The size of the emitting region, $r$, is either measured directly by means of inteferometric observations, or is deduced from the time scale $\Delta t$, as indicated above. The magnetic field, $B$, is measured or bounded from above according to the presence or 
absence of a synchrotron self-absorption turn-over at low frequencies. The energy distribution of the relativistic electrons and their total number, $N$, follow from the spectral distribution and total luminosity of the radio emission, respectively. The self-consistent solution for the overall spectrum is a pure synchrotron spectrum only in the limit of a very dilute source. In the opposite limit of a very compact source one has a synchrotron-self-Compton spectrum (SSC): here a cascade of Compton components is included, produced in succession by the same relativistic electrons which emit the "seed" synchrotron photons. The parameter governing the relative importance of any two successive orders is

$$
A=\sigma_{T} N \gamma^{2} / r^{2}
$$

where $\gamma$ is a suitable average of the particle Lorentz factor. The luminosity contributed by the $i$-th element in the cascade is

$$
L_{i} \approx C B^{2} r^{2} A^{i+1}
$$

which includes the synchrotron luminosity, $L_{s}$, for $i=0$. When $A$ is larger or smaller than unity, the overall luminosity is dominated by the high or low frequency emission, respectively. In radio astronomical terms the same notion is expressed by means of the synchrotron brightness temperature, $T_{b}$ : A being larger or smaller than unity is equivalent to $T_{b}$ being larger or smaller than a critical value $T_{C} * 10^{12}{ }^{\circ} \mathrm{K}$ (Kellermann and Pauliny-Toth 1969). If SSC is relevant to the continuum of AGN's, then the flatness illustrated in Fig. I indicates an effective brightness temperature close to the critical value.

Fast variations around a given radio luminosity imply small $r$ 's and large apparent $T_{b}$ 's; these in turn imply large luminosities at higher frequencies - typically in the $X$-ray range - , in contradiction with the luminosities which are actually observed. Bulk motions at relativistic speeds (Rees 1967) allow large $r$ 's for the same $\Delta t^{\prime} s$, and entail an effective $T_{b}$ arbitrarily lower than the apparent one.

We are thus led to the conclusion that relativistic flows are an essential ingredient of $A G N$ electrodynamics, and that the conditions are suitable for the SSC process to play an important role.

From a theoretical point of view SSC is appealing since it naturally produces a wide-band spectrum, without postulating a wide-band distribution of radiators. Also, it always comes with compact synchrotron sources, and avoids the introduction of further ad hoc hypotheses.

\section{POWER SUPPLY VIA POYNTING FLUX}

In the preceding arguments the magnitude of the relativistic effects depends on the Doppler factor, $D$, of the bulk motion relative to the observer; even if a universal value of the Lorentz factor, $T$, were enforced by some acceleration mechanism, a variety of line-of-sight angles would still result in a variety of $D^{\prime} s$; there is no reason why several apparent brightness temperatures and several Doppler factors should conspire so as to produce a rather narrow distribution of effective brightness temperatures, clustered around 
$T_{c}$. One is led to the conclusion that some kind of universal radiation process is at work in the proper frame of the emitting plasma, endowed with a selfregulating mechanis $m$ which keeps $T_{b}$ at around $T_{c}$. Once the luminosity ratios among the various spectral domains have been set at around unity in the proper frame, an arbitray Lorentz transformation to the observer frame leaves them unchanged, and only affects the variability time scales.

In our formalis $m$ the relative weight of the spectral crders is given by $A$, defined in $E q .1$. When $A=1, E q$. 2 shows that the value of the luminosity common to all orders is $L_{B}=c B^{2} r^{2}$, that is, the energy density in magnetic field flowing across the source boundaries at speed $c$. The primary power supply appears to be in the form of electromagnetic fields, which are converted with high efficiency into observable radiation.

A plausible scenario where these conditions could be met has been suggested by Pacini and Salvati (1978): they assumed that the active region is energized by large scale electromagnetic fields with $E \approx B, E \perp B$, so that the Poynting vector flux $\approx C B^{2} r^{2}$. The electromagnetic power is spent in maintaining a suitable number of electrons at relativistic energies, at balance with their radiative losses; the gains-vs-losses balance guarantees a high radiative efficiency, and the overall luminosity has to be equal to $L_{B}$.

Furthermore, when the process is roughly half-way, the relativistic electrons see a comparable amount of energy density in magnetic field and target synchrotron photons. Hence we have

$$
\begin{aligned}
& L_{s} /\left(c r^{2}\right) \approx B^{2}, \quad L_{s} /\left(c r^{2}\right) \approx N \quad c \quad \sigma_{T} \gamma^{2} B^{2} /\left(c r^{2}\right)=A B^{2} \\
& A \approx 1
\end{aligned}
$$

and this is the condition required for the individual orders to be comparable to one another.

In the following we will show how the proposed scheme is consistent with global pictures currently proposed for the central regions of Active Galactic Nuclei; it is also amenable to a quantitative description, provided that a number of approximations are accepted; and, finally, it can be proven very easily to be Lorentz-invariant.

\section{A MODEL}

According to recent studies of AGN continua, several different processes are at work simultaneously (Malkan and Sargent 1982; Netzer 1985). Very crudely, one can distinguish a thermal bump, which is responsible for most of the ionizing radiation, and is related to at least part of the X-rays via nonrelativistic comptonization; this is superimposed on a power law, presumably of a non-thermal origin, which runs from the submillimeter region to the hard $X$-rays; the radio emission in radio loud objects is an extension of this power law, coming from larger and less compact regions, and part of the infrared might be due to reprocessing by dust.

The physical picture is similarly complicated (e.g., Rees 1984). While the prime mover is generally identified with a mass-accreting potential well, different components are thought to be relevant to different parts of the spectrum. The UV bump and the thermal $X$-rays are probably due to an accretion disk and its corona. If a magnetic field threads the disk, or the disk 
and the black hole horizon (Blandford 1976; Blanford and Znajek 1977), part of the power could be extracted by electromagnetic stresses, rather than viscous stresses; in this case the vicinity of the hole will be pervaded by large scale electromagnetic fields organized in a wind-like geometry, suitable - in our scenario - for the production of the non-thermal powerlaw. The wind would be eventually collimated, perhaps by magnetic pinch, and at the appropriate distance it would become observable in the radio domain.

Close to the black hole, at distances sufficiently smaller than the scale of the collimating processes, the geometry is expected to resemble the far zone of an aligned "pulsar" (Goldreich and Julian 1969). An artist's view of the proposed arrangement is given in Fig. 2. A dipolar poloidal magnetic field induces a quadrupolar charge distribution, which sustains a quadrupolar poloidal electric field. The charges move radially outward at practically the speed of light, and generate currents which sustain a toroidal magnetic field having opposite signs in the two hemispheres. Since the charges are moving
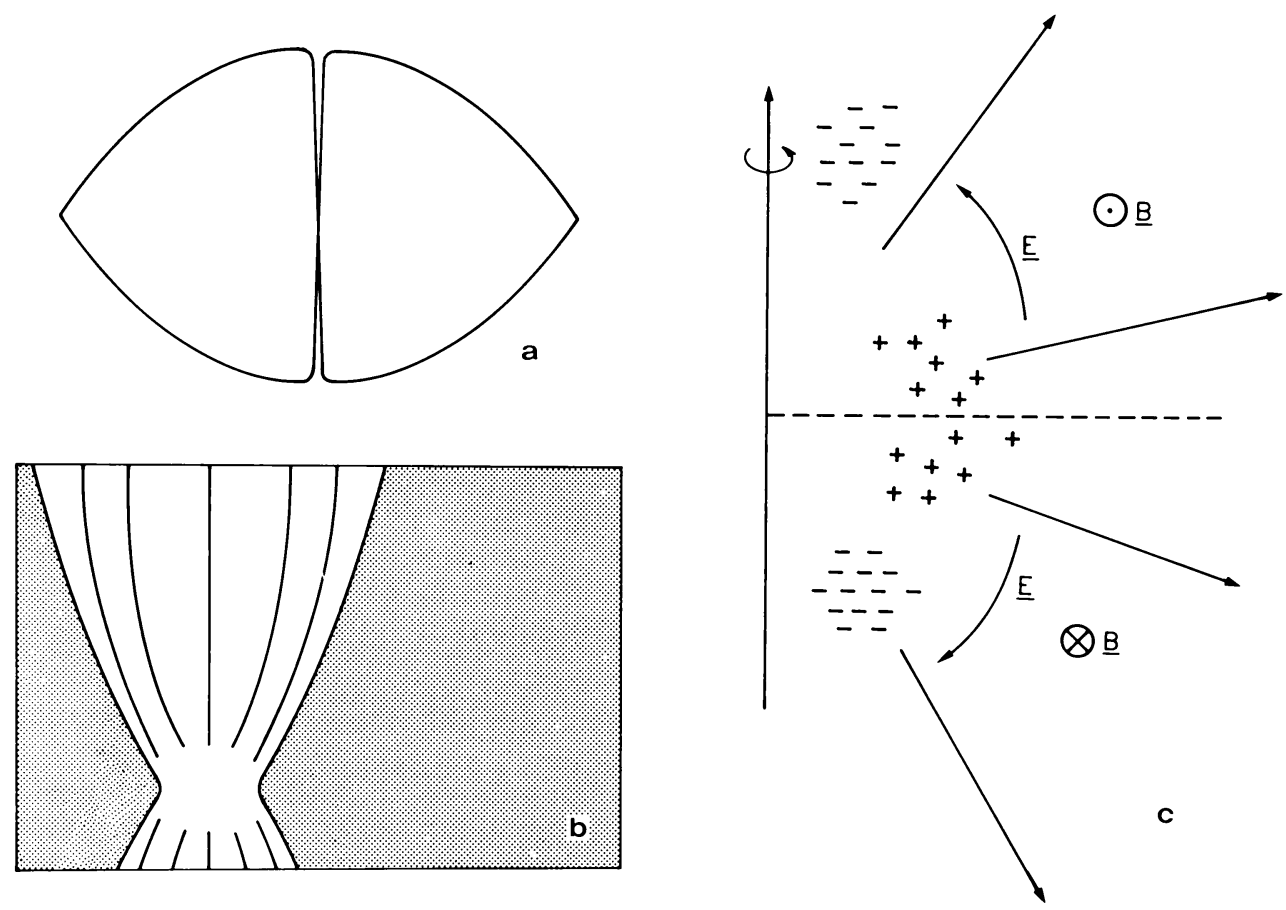

Figure 2. Schematic diagram indicating location and geometry of the proposed non-thermal source. Panels $a, b$, and $c$ are a zooming sequence towards the innermost AGN regions. They show respectively: a thick accretion disk; the region of funnel formation; the field and charge arrangement in a relativistic wind. From Calvani and Nobili (1983), Phinney (1983), and Goldreich and Julian (1969), with modifications. 
with speed $\approx C$, the $E$ and $B$ fields are very nearly equal

$$
P_{e} \approx E / r, \quad j \approx c P_{e} \approx c E / r, B \approx r j / c \approx E
$$

Then the drift velocity is close to $c$ and is directed radially outward, and an approximate dynamical consistency is ensured. The large scale electromagnetic fields make the dominant contribution to the energy density and the energy flux. The carriers are irrelevant as far as the global energetics are concerned, and their nature and total number cannot be determined without including the boundary conditions at injection and solving the acceleration problem. For the sake of simplicity, and in agreement with previous suggestions, we consider a plasma composed solely of electrons and positrons.

Our basic assumption is that the radial outflow of the particles is somehow perturbed. One can imagine "collisions" with heavy "scattering centers", or with the waves of a turbulent substratum. We postulate that the effect of such collisions is to remove momentum without changing the energy; the momentum removal is described by means of a scale length, $S$, which enters the momentum equation via a term $\mathrm{m} \mathrm{c}^{3} / \mathrm{s}$. The collisions decrease the bulk velocity of the outflow, and the surplus energy goes into particle gyrations; these can be relativistic, with Lorentz factor $\gamma$. Since the $\mathbf{v} \times \mathbf{B}$ force is decreased, the $E$ force brings opposite charges together in the meridian plane; this means closing some current loops, and $E$ and $B$ diminish together; the bulk motion is accelerated anew because of the drift along $E$ and equilibrium is reestablished at a lower value of the Poynting vector flux. Of course the process goes on continuously, and the energy which is being fed into disordered particle motions is available for radiation. The details of the formalism will be given elsewhere; here we list the free parameters of the model, and indicate the - potentially - observable quantities which depend on them.

At a fiducial radius $r_{0}$ one has to assign: i) the fields $E_{0}$ and $B_{0}$ : these determine the available power supply $\subset E_{0} B_{0} r_{0}^{2}$, the drift velocity $C E_{0} / B_{0}$, and the bulk Lorentz factor $T$ corresponding to the latter; ii) the particle number density $n_{0}$ : this determines the density profile via particle number conservation, $n=n_{0}\left(r_{0} / r\right)^{2}$, and the Thomson depth $\tau=n_{0} r_{0} \sigma_{T}$; the qyantities $S_{0}$ and $k$, describing the behaviour of $S$ with distance, $S=S_{0}\left(r_{0} / r\right)^{k+1}$ : these concur in the determination of the efficiency $\varepsilon$, as shown in $E q .6$ below. Then, the consumption of the electromagnetic field is described as follows

$$
\begin{aligned}
& d y^{2} / d x=-\sigma x^{-k-1}, \quad x=r / r_{0}, \quad y=r B /\left(r_{0} B_{0}\right) \\
& \sigma=4 \pi m c^{2} n_{0} r_{0} T^{2}\left(1-1 / T^{2}\right)^{\frac{1}{2} /\left(S_{0} B_{0}{ }^{2}\right)}
\end{aligned}
$$

By integrating up to infinity, one easily finds the radiative efficiency

$$
\varepsilon=\sigma / k
$$

The comoving Lorentz factor $\gamma$ is a function of position, and is determined by the requirement that the energy input - depending on $S$ - be equal to the radiative losses. An exact solution involves a careful treatment of the 
transfer problem; order-of-magnitude wise, however, one finds

$$
\gamma \approx \Gamma(\varepsilon / \tau)^{\frac{1}{2}}
$$

When $\varepsilon$ approaches unity, $\nu F_{\nu}$ becomes flat, as expected. Simple arguments can be given showing that this result is indeed independent of $\Gamma$ : the inverse Compton emission is proportional to $\mathrm{I}^{-4}$ because of the angular factor introduced by the relativistic expansion, but the synchrotron emission $L_{s}$ has the same dependence. Very high $\Gamma$ "s require $E$ and $B$ fields very nearly equal, and the residual B field in the comoving frame is very small; then, taking advantage of the Lorentz invariance of the radiated power, one can write $L_{S} \propto(\gamma B / T)^{2}=(\gamma \Gamma)^{2} B^{2} \Gamma^{-4}$, which proves our assertion, since $\gamma \Gamma$ is the particle Lorentz factor in the observer frame.

\section{CONCLUSIONS}

Besides the general notions of relativistic velocities and SSC, the comparison between our model and radio observations of AGN's is rather difficult. It is plausible that the radio emitting plasma is the same plasma of the wind which we have been discussing, but the radio emission takes place at such a large distance that a good deal of extrapolation is needed to link to the latter the properties measured in the former.

However, as the observing frequency increases and approaches the submillimeter range, the emitting region comes closer and closer to the center. In the pictorial language of the introduction, increasing the observing frequency is equivalent to going down the "chimney" toward the real "fire". There is evidence, not only from preliminary synthetic spectra, but also from direct observations (Owen et al. 1981), that at the highest radio frequencies one already touches the tip of the core power law. It is obvious that systematic studies of this spectral region, including perhaps VLBI mapping, would directly affect our knowledge of the "fire", and would put on a firmer footing theories as the one presented here.

\section{REFERENCES}

Begelman, M.C., Blandford, R.D., and Rees, M.J. 1984, Rev. Mod.Phys., 56,255

Blandford, R.D. 1976, M.N.R.A.S., 176, 465

Blandford, R.D., McKee, C.E., and Rees, M.J. 1977, Nature, 267, 211

Blandford, R.D., and Znajek, R.L. 1977, M.N.R.A.S., 179, 433

Calvani, M., and Nobili, L. 1983, in Astrophysical Jets, eds. A. Ferrari and A.G. Pacholczyk (Dordrecht: Reidel), p. 189

Cohen, M.H., and Uriwin, S.C. 1984, in $V L B I$ and Compact Radio Sources, IAU Symp. no. 110, eds. R. Fanti, K. Kellermann, and G. Setti

(Dordrecht: Reidel), p. 95

Goldreich, P., and Julian, W.H. 1969, Ap. J., 157, 869

Kellermann, K.I., and Pauliny-Toth, I.I.K. 1969, Ap.J. (Letters), 155, L71 
Malkan, M.A., and Sargent, W.L.W. 1982, Ap. J., 254, 22

Netzer, H. 1985, Ap. J., 289, 451

Owen, F.N., Helfand, D.J., and Spangler, R.S. 1981, Ap.J.(Letters), 250, L55

Pacini, F., and Salvati, M. 1978, Ap. J. (Letters), 225, L99

Padrielli, L. 1984, in VLBI and Compact Radio Sources, IAU Symp. no. 110 , eds. R. Fanti, K. Kellermann, and G. Setti (Dordrecht: Reidel), p. 169

Phinney, E.S. 1983, Ph.D. Thesis, University of Cambridge.

Rickett, B.J. 1986, Ap.J., 307, 56

Rees, M.J. 1967, M.N.R.A.S., 135, 34

Rees, M.J. 1984, Ann. Rev. Astr. Ap., 22, 472

Rybicki, G.B., and Lightman, A.P. 1979, Radiative Processes in Astrophysics (New York: Wiley) 